\title{
Reparación de cardiopatía congénita crítica en el prematuro
}

\author{
Repair of critical congenital heart disease in prematurity \\ Karen Mármol Realpe, ${ }^{*}$ Humberto García Aguilar, ${ }^{* \neq}$ Eduardo Benadón Darszón, ${ }^{\neq}$ \\ Pablo Tietzsch Escalante, ${ }^{\ddagger}$ Antonio Benita ${ }^{\S}$ \\ Citar como: Mármol RK, García AH, Benadón DE, Tietzsch EP, Benita A. Reparación de cardiopatía congénita \\ crítica en el prematuro. Acta Med. 2021; 19 (1): 123-125. https://dx.doi.org/10.35366/98581
}

\section{Resumen}

Las cardiopatías congénitas críticas en el periodo neonatal son condiciones que presentan alta mortalidad, siendo mucho más graves cuando se asocian a un recién nacido prematuro y peso menor de 2,000 gramos. Estas lesiones son dependientes del conducto arterioso, por lo que el diagnóstico temprano y el uso de prostaglandinas para mantener la vida y la estabilidad hemodinámica permiten llevar a la reparación quirúrgica a un paciente lo más estable posible. Presentamos el caso clínico de un recién nacido prematuro de 1,700 gramos y coartación aórtica crítica, a quien se llevó a reparación mediante coartectomía extendida, con una evolución satisfactoria a pesar de las variables de riesgo de edad gestacional y peso. Presentamos los recursos diagnósticos de imagen para confirmación del diagnóstico y resolución quirúrgica. El manejo en cuidados intensivos es fundamental para los buenos resultados como en nuestro caso, y discutiremos brevemente el papel del tamiz cardiaco neonatal en este grupo de patologías.

Palabras clave: Cardiopatía congénita crítica, recién nacido prematuro, coartación aórtica.

\section{INTRODUCCIÓN}

Los defectos cardiacos son las malformaciones más frecuentes al nacimiento, se estima a nivel mundial una incidencia de 10-12 niños por cada 1,000 nacidos vivos. ${ }^{1}$ Esto implica que, si en nuestro país hay un poco más de 2 millones de

\section{Abstract}

Critical congenital heart diseases in the neonatal period are conditions that present high mortality, being much more serious when associated with a premature newborn and weighing less than 2,000 grams. These lesions are dependent on the ductus arteriosus, so early diagnosis and the use of prostaglandins to maintain life and hemodynamic stability allows a patient to be as stable as possible for surgical repair. We present the clinical case of a premature newborn weighing 1,700 grams with critical aortic coarctation, who underwent repair through extended coartectomy, with a satisfactory evolution despite the risk variables of gestational age and weight. We present the diagnostic imaging resources for confirmation of the diagnosis and surgical resolution. Management in intensive care is essential for good results, as in our case, and we will briefly discuss the role of neonatal cardiac screening in this group of pathologies.

Keywords: Critical congenital heart disease, premature newborn, aortic coarctation.

* Departamento de Cardiología Pediátrica del Centro Médico Nacional 20 de Noviembre, ISSSTE. CDMX, México.

₹ Médicos Especialistas en Pediatría, S.A. de C.V. Hospital Ángeles Lomas. CDMX, México.

$\S$ Departamento de Cirugía Cardiovascular del Instituto Nacional de Cardiología "Ignacio Chávez". CDMX, México.

Correspondencia:

Humberto García Aguilar

Correo electrónico: humgaa@yahoo.com

Aceptado: 10-09-2020.

www.medigraphic.com/actamedica 


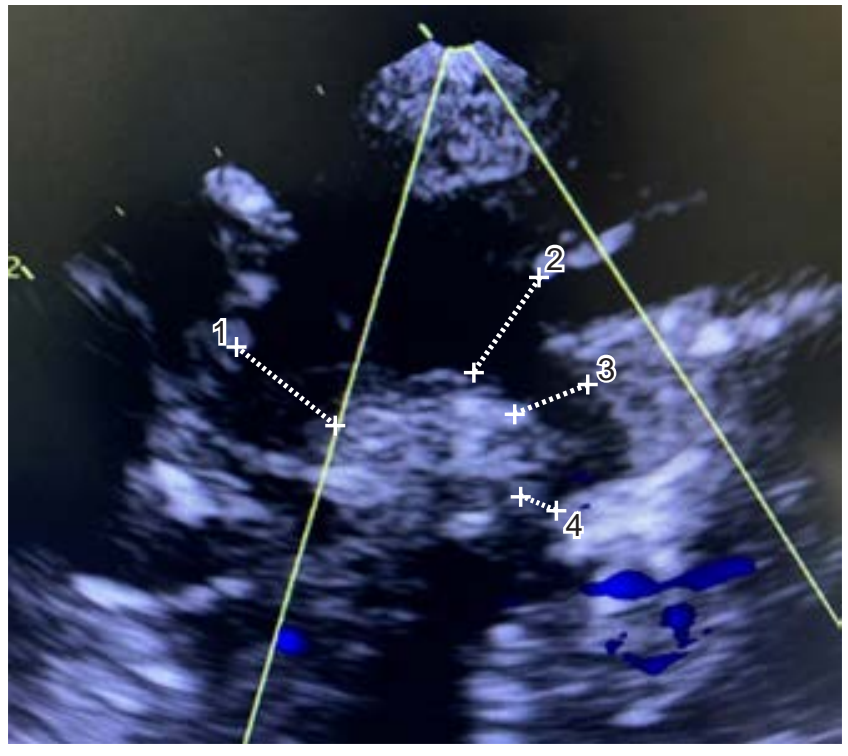

Figura 1: Ecocardiograma bidimensional transtorácico en el que se demuestra coartación aórtica yuxtaductal crítica con un diámetro de $1.5 \mathrm{~mm}$. Medidas: aorta ascendente $6 \mathrm{~mm}$, aorta transversa $4 \mathrm{~mm}$, descendente $3 \mathrm{~mm}$, istmo $1.5 \mathrm{~mm}$. El gradiente medio $52 \mathrm{mmHg}$ a nivel del istmo.

\section{CASO CLÍNICO}

Presentamos el caso de un recién nacido de embarazo gemelar monocoriónico, producto de la cuarta gestación y nacimiento a las 35.2 semanas. La indicación de la cesárea fue por alteración en el crecimiento intrauterino del gemelo 2 (peso estimado por USG un día previo; gemelo 1: 2.4 $\mathrm{kg}$, gemelo 2: $1.7 \mathrm{~kg}$ ) y flujos placentarios normales. Se obtiene a recién nacido por cesárea, gemelo 2 con llanto vigoroso y esfuerzo respiratorio adecuado. Valoración Apgar 8-8, peso 1,700 gramos, escala de Silverman 2 y frecuencia respiratoria de 65 respiraciones por minuto, manejado con oxígeno indirecto con casco cefálico a $40 \%$ y $5 \mathrm{~L} / \mathrm{min}$. Inicialmente asintomático con pulsos presentes las primeras 12 horas de vida extrauterina. Posteriormente muestra soplo sistólico en mesocardio con irradiación al dorso. Los pulsos arteriales se encontraban presentes en las cuatro extremidades, aunque los femorales discrepan de los radiales en intensidad y amplitud. El ecocardiograma inicial mostró coartación aórtica crítica (CoAo) con gradiente medio de $52 \mathrm{mmHg}$, hipoplasia del arco aórtico y ductus arterioso permeable grande (Figura 1). El abordaje inicial fue la infusión de alprostadil (Alisitin $500^{\circledR}$ ) a una dosis $0.1 \mu \mathrm{g} / \mathrm{kg} / \mathrm{min}$, retiro del aporte de oxígeno y solicitud de angiotomografía de aorta (previa valoración de una función renal normal) donde se confirma el diagnóstico con descripción de coartación aórtica con diámetros: ascendente
$6 \mathrm{~mm}$, transversal $5 \mathrm{~mm}$, descendente $4 \mathrm{~mm}$ y zona de coartación $2 \mathrm{~mm}$ (Figura 2).

Se decidió llevar a corrección quirúrgica urgente con las consideraciones de riesgo por edad y peso. Mediante abordaje por toracotomía lateral izquierda se realizó coartectomía ampliada, sección y sutura del ductus arterioso sin complicaciones (Figura 3).

El paciente se manejó en la Unidad de Cuidados Intensivos Neonatales (UCIN) del Hospital Ángeles Lomas, lográndose una extubación temprana, evolución estable, con nutrición mixta y sin necesidad de apoyo inotrópico.

Los controles ecocardiográficos postoperatorios mostraron un gradiente medio residual de $12 \mathrm{mmHg}$, se logró egreso a casa una vez alcanzado un peso de 2,000 g.

\section{DISCUSIÓN}

Las cardiopatías congénitas críticas en el periodo neonatal son lesiones obstructivas que comprometen la circulación sistémica o pulmonar con alta mortalidad debido a que dependen del ductus arterioso (DA), el cual es un defecto obligado para mantener la vida del recién nacido. Si no se logra diagnosticar la anomalía cardiaca antes del cierre fisiológico del DA, el colapso circulatorio posterior compromete a otros órganos llevando a la muerte al paciente o en su caso, su posterior recuperación ocasiona mayores

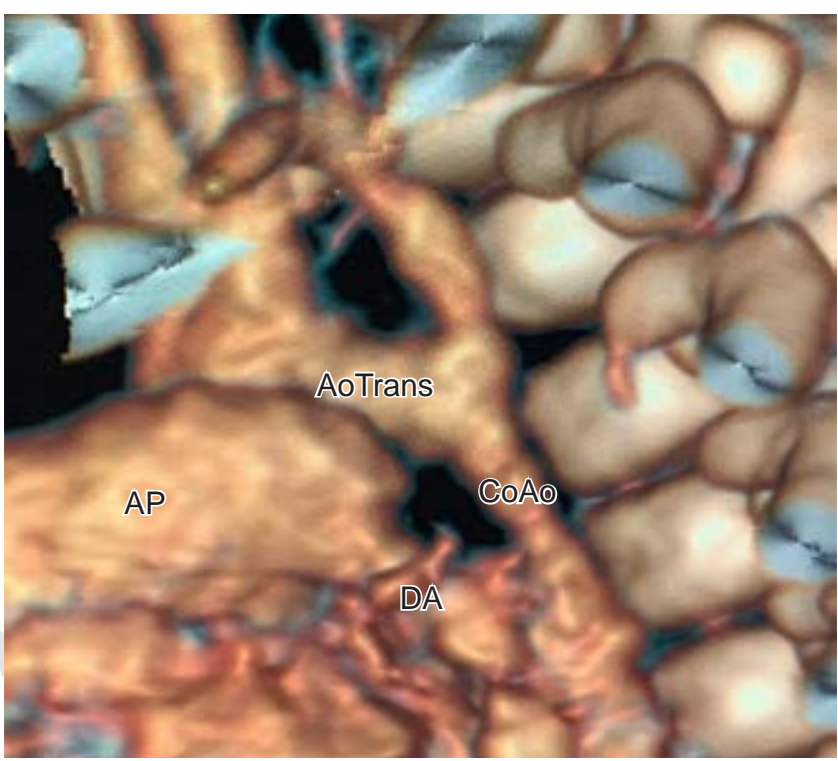

Figura 2: Reconstrucción en 3D de angiotomografía donde se observa hipoplasia de aorta descendente y coartación aórtica con diámetro de $2 \mathrm{~mm}$.

AoTrans $=$ aorta transversa, $\mathrm{AP}=$ arteria pulmonar, $\mathrm{DA}=$ ductus arterioso, $\mathrm{CoAo}=$ coartación aórtica. 


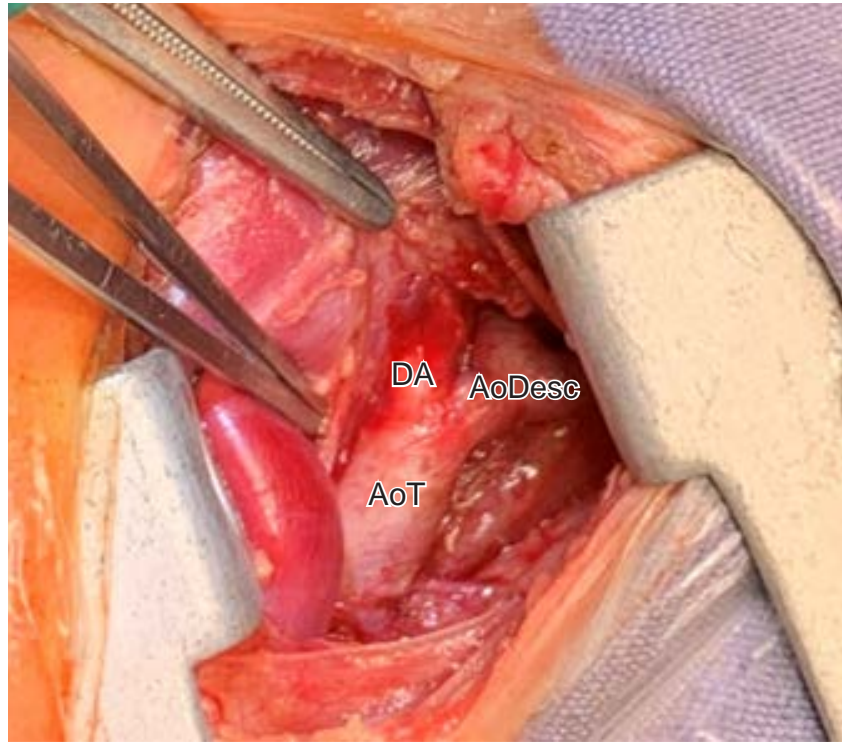

Figura 3: Coartectomía lateral izquierda en la que se aprecia el ductus arterioso (DA) del tamaño de la aorta torácica (AoT) y la zona de hipoplasia de la aorta descendente (AoDesc).

complicaciones después de la reparación. Por lo anterior, el pronóstico de supervivencia de los recién nacidos con lesiones cardiacas críticas depende de evitar el cierre del DA con el uso de prostaglandinas para prevenir el choque cardiogénico secundario y llevar a cirugía a un paciente estable.

En relación con los embarazos múltiples, una publicación reciente reporta que las lesiones congénitas cardiacas en embarazos gemelares monocoriónicos se asocian a un riesgo $47 \%$ mayor en comparación con embarazos únicos, ${ }^{3}$ lo cual, considerando el riesgo habitual que se encuentra entre $1-4 \%$, no es un incremento significativo. ${ }^{4}$ Lopes y colaboradores describieron en un estudio de población de gemelos un riesgo de 3.1 mayor (con intervalo de confianza 95\% 1.5-6.4) de presentar coartación aórtica neonatal en esta población, ${ }^{5}$ lo que representa una patología a considerar en un recién nacido gemelar con choque cardiogénico temprano.

Finalmente, hay necesidad de manejo y reparación en el periodo neonatal de la lesión cardiaca y se tendrán que considerar las condiciones que incrementen el riesgo de muerte entre los pacientes. La mortalidad es hasta dos veces mayor en los prematuros, puesto que el bajo peso al nacer y la presencia de asfixia tienen gran impacto en la supervivencia. ${ }^{6}$ En general en las enfermedades cardiacas congénitas graves la supervivencia en el primer año de vida fluctúa de 62.8 a $79.9 \%$. Antes de la cirugía cardiaca sólo $30 \%$ de los niños con enfermedades cardiacas congénitas severas sobrevivió hasta la edad adulta. ${ }^{7}$

\section{Importancia del tamiz cardiaco neonatal}

Se ha informado que 30\% de los casos de cardiopatías congénitas críticas se diagnostican después de los tres días del nacimiento en los Estados Unidos y 25\% se diagnostica después del alta en el norte de Inglaterra. Por esta razón la recomendación del examen de oximetría de pulso se ha extendido mundialmente, debiendo realizarse de forma rutinaria en todos los recién nacidos entre las 24 y 36 horas del nacimiento. ${ }^{7}$ Esta herramienta de fácil aplicación, no invasiva y de bajo costo debe considerarse obligatoria en todos los hospitales donde nacen niños. ${ }^{8}$

El éxito de nuestro caso presentado, a pesar de tener factores de riesgo como la edad gestacional y el peso, fue el diagnóstico oportuno y llevar a corrección a un paciente estable.

\section{REFERENCIAS}

1. Mendieta-Alcántara GG, Santiago-Alcántara E, Mendieta-Zerón H, Dorantes-Piña R, Ortiz de Zárate-Alarcón G, Otero-Ojeda GA. Incidencia de las cardiopatías congénitas y los factores asociados a la letalidad en niños nacidos en dos hospitales del Estado de México. Gac Med Mex. 2013; 149 (6): 617-623.

2. Márquez-González H, Yáñez-Gutiérrez L, Rivera-May JL, LópezGallegos D, Almeida-Gutiérrez E. Análisis demográfico de una clínica de cardiopatías congénitas del Instituto Mexicano del Seguro Social, con interés en el adulto. Arch Cardiol Méx. 2018; 88 (5): 360-368. doi: 10.1016/j.acmx.2017.09.003

3. Herskind AM, Almind Pedersen D, Christensen K. Increased prevalence of congenital heart defects in monozygotic and dizygotic twins. Circulation. 2013; 128 (11): 1182-1188. doi: 10.1161/ CIRCULATIONAHA.113.002453.

4. Martínez-Quintana E, Romero-Requejo A, Rodríguez-González F. Cardiopatías congénitas y embarazo. Clin Invest Gin Obst. 2016; 43 (1): 24-31. http://dx.doi.org/10.1016/j.gine.2014.11.002

5. Lopes SAVDA, Guimarães ICB, Costa SFO, Acosta AX, Sandes KA, Mendes CMC. Mortality for critical congenital heart diseases and associated risk factors in newborns. A cohort study. Arq Bras Cardiol. 2018; 111 (5): 666-673. doi: 10.5935/abc.20180175.

6. Chu PY, Li JS, Kosinski AS, Hornik CP, Hill KD. Congenital heart disease in premature infants 25-32 weeks' gestational age. J Pediatr. 2017; 181: 37-41.e1. doi: 10.1016/j.jpeds.2016.10.033.

7. Wong KK, Fournier A, Fruitman DS, Graves L, Human DG, Narvey M et al. Canadian Cardiovascular Society/Canadian Pediatric Cardiology Association position statement on pulse oximetry screening in newborns to enhance detection of critical congenital heart disease. Can J Cardiol. 2017; 33 (2): 199-208.

8. Cullen BPJ, Guzmán CB. Tamiz de cardiopatías congénitas críticas. Recomendaciones actuales. Acta Med. 2014; 12 (1): 24-29. 